\title{
What is and What is not the Digital Earth?*
}

\author{
Eugene Eremchenko ${ }^{[0000-0002-4416-7617]}$ \\ Lomonosov Moscow State University, Moscow GSP-1, 119991, Russia \\ eugene.eremchenkolgmail.com
}

\begin{abstract}
The article addresses to a fundamental issue facing the concept of the Digital Earth in 2020 - what is the definition of the Digital Earth? 22 years after the announcement of the concept and 15 years after its first mass and successful implementation in the service Google Earth in 2005 this question remains unanswered. In the article the current status of the development of the Digital Earth concept by 2020 is considered, a methodology for the definition of the Digital Earth, based on the classification of the diversity of existing geospatial approaches and the identification of key factors that provide its unique functionality, is proposed and discussed. Distinction between the Digital Earth and others geospatial approaches is provided. The intrinsic connection of definition and classifications is grounded, the new definition of the Digital Earth and complimentary classification of geospatial methods are offered. Semiotics aspects of the Digital Earth are discussed briefly. The question of the eligibility of technologies and systems to the Digital Earth is considered. The perspective issues of further development of the Digital Earth are considered.
\end{abstract}

Keywords: Digital Earth, Visualization, Typology, Cartography, Semiotics.

\section{Introduction}

In 2020 the Digital Earth initiative, first introduced by U.S. Vice President Albert Gore in 1998, turned 22 years old. Digital Earth was being promoted as a new, revolutionary approach to handling geospatial information with fabulous functionality, and as a core of the new smart response and effective governance policy that will offer many benefits to all mankind. In his well-known speech [1] Gore envisioned and highlighted two milestone years in future development of new concept -2005 and 2020: "What we will be able to do in 2005 will look primitive compared to the Digital Earth of the year 2020".

The prediction appeared to be remarkable. The year 2005 was indeed a turning point in the development of the Digital Earth and marked the beginning of the "geospatial revolution" with the unveiling of Google Earth [2] - the first service that fully

Copyright (C) 2020 for this paper by its authors. Use permitted under Creative Commons License Attribution 4.0 International (CC BY 4.0).

* Supported by RSF grant \#20-47-01001 (data analysis, investigations, discussion and conclusions) and by RFBR grant \#18-05-00236 (selections and review of the raw materials). 


\section{E. Eremchenko}

embodied this concept and gained extreme popularity. Until now, Google Earth remains the most striking implementation of the ideas behind the Digital Earth concept. In 2020, as Gore presumed, the Digital Earth should go far ahead, and the achievements of 2005 against its background should seem primitive. Today, in 2020, we have an opportunity to check the accuracy of the second part of his forecast, but the situation looks ambiguous. In 2020, the current status of the concept of the Digital Earth seems to be at least contradictory. That is the reason why the year 2020 is particularly suitable for checking the current status of the development and implementation of the Digital Earth concept and defining plans for the future.

On the one hand, the geospatial revolution has become a fact of our life and had an irreversible impact on all mankind. Now the Digital Earth is officially recognized throughout the world and has already established itself as a best approach for working with geospatially located data. Digital Earth has gained huge popularity and widely implemented with remarkable results. Google Earth, the clearest example of the new concept, was downloaded more than billion of times six years after its launch [3]. One year after the launch of the Google Earth, in 2006, the International Society for Digital Earth (ISDE) was established [4]. 11 Digital Earth Symposiums [5] and 7 Digital Earth Summits [6] were held around the world up to this moment - the largest and most authoritative international forums dedicated to discussing the scientific paradigm, technological requirements, social benefits and outreach of the concept within rich interdisciplinary context. ISDE scientific activity is concentrated around the wellrecognized journals - the International Journal of Digital Earth (IJDE) with 2019 impact factor 3,097 [7] and his sister journal Big Earth Data (since 2018) [8] - both published by the Taylor\&Fransis Group. ISDE becomes a member of world leading scientific organizations - like International Science Council (ISC), United Nation Committee of Experts on Global Geospatial Information Management -), United Nation Committee of Experts on Global Geospatial Information Management - Geospatial Societies (UN-GGIM GS), Committee on Data of the International Science Council (CODATA), etc.

The development of ISDE received a new impetus in mid-2010s with the start of major infrastructure projects directly inspired by the Digital Earth concept and organized under the umbrella of ISDE. China, Australia [9] and Russia [10] have recognized the Digital Earth as the basis for national geospatial data policies. It's worth mentioning Digital Silk Road Alliance (DSRA) project, launched in 2016 as a informational backbone of the Road and Belt initiative that covering virtually all of Eurasia, and the Digital Earth Australia project, launched in 2017 and resulting in a rapid transformation of usual practices of working with geospatial data. For example, one year after the launch of the project Digital Earth Australia, production of official paper topographic maps were completely cancelled in that country [11]. Two weeks after its recognition in Australia, Digital Earth was declared as the basis for national policy on space remote sensing in Russia as well.

On the eve of 2020, the Manual of Digital Earth [12] - the first detailed and comprehensive summary of this concept at its current level of development - was published and shared as open source edition. An open access publication of 852 pages, which outlined the knowledge accumulated to date in the field of Digital Earth and the experience of its use in solving a variety of tasks, has gained amazing popularity in the first half-year, it was downloaded more than half a million times. 
In 2020 the implementation of Digital Earth has become a mandatory requirement for quality management. The coronavirus pandemic COVID-19 has shown the extreme need of humankind to ensure sustainable development and to implement the Digital Earth as a state-of-the-art decision making support environment due to its global extent and scale-less nature. The need for the Digital Earth as a highly effective tool to support global decision making has reached an unprecedented level.

On the other hand, scientific and technological progress between 2005 and 2020 has been far more modest than expected 22 years ago. There is a visible and growing contradiction between, from one hand, the urgent need for the Digital Earth and sustained technological advance - and, from another hand, small and controversial scientific developments in this field. Google Earth still remains the best embodiment of the Digital Earth concept 15 years after its launch, even though its functionality has been severely limited and development has virtually ceased [13]. The Digital Earth is developing in many ways now in an ad hoc manner. Until now, there is no Digital Earth standard, and even no ultimate basis for its developing - the comprehensive updated and widely accepted scientific definition of the Digital Earth. The problem looks so pressing that in 2020 ISDE held a special meeting on the issue of developing a scientific definition of the Digital Earth.

The lack of definition does not allow to separate the Digital Earth from the variety of its analogues in the current situation of rapid emergence of new geospatial products and solutions, which can be difficult to attribute to any particular class. Thus, the discussion of the current status and prospects of the concept rises the question: what is and what is not the Digital Earth? Where are the limits of the Digital Earth?

Obviously, the definition and classification issues are interdependent - definition provides classification, and vice versa. The resolution of these issues is particularly important now, since the term "Digital Earth" is often understood in a hardly acceptable broad sense. There is a clear tendency to consolidate under the umbrella of the Digital Earth everything related to geospatial information, including all components of the process of creation, storage and use of localized data - remote sensing satellites, communications and navigation, data storages and transmission lines, hardware complexes and algorithms, mobile applications, etc. This approach, however, excludes discussion of the obvious novelty of the Digital Earth, because all information systems are now digital and all information is localized in geospace; it appears that there is nothing outside the Digital Earth that is obviously wrong. It is therefore important to undertake a comparative analysis, to identify and compare approaches and methods that are obviously not relevant to the Digital Earth.

Discussing the challenges in the development of the concept of the Digital Earth is particularly important in the context of 2020. Beginning with the second decade of the 21 st century, ISDE began to formulate a scientific agenda for the coming decade. The first declaration Google Earth Vision 2020 was developed in 2011 [14]. The task today is to develop a similar program, Digital Earth Vision 2030, which should address the problems that have emerged so far. Definition of Digital Earth and recognizing its place amongst the whole variety of the geospatial approaching became core problem of the current stage of the development of the concept. 


\section{E. Eremchenko}

\section{Methods and research}

Many attempts have been made to define the Digital Earth, but Gore's very first definition of the Digital Earth still appears to be the most successful and effective. He defined the Digital Earth as "... a multi-resolution, three-dimensional representation of the planet, into which we can embed vast quantities of geo-referenced data" [1]. Indeed, the Digital Earth supports different scales of representation of scene and is three-dimensional, i.e. it allows viewing a scene from different angles. However, Gore's definition needs some clarification.

From the point of view of the common user perception, the answer to the question "what is the Digital Earth" is quite simple - it's a generic term for a variety of products similar to Google Earth. From a historical point of view, this issue also hardly raises the questions - this is exactly the kind of clearly recognizable product was anticipated in literature many decades before our era. The most striking example is, probably, the magical "Woland's Globe" from Mikhail Bulgakov's novel "The Master and Margarita" [15].

Understanding what is the Digital Earth equals to understanding what is not the Digital Earth. In turn, separation of these too classes produces the classification of whole variety of the similar approaches within their specific domain and to identify the relationships between them. To answer this question, it is necessary to define the domain within which the problem should be solved, and identify its counterparts. We can assume that this domain is geospatial visualization, because the perception of space should be visual and, accordingly, can only be provided by means of visualization. Within this domain, there are a variety of approaches to the representation of data - maps, globes, atlases, GIS, 3D-models, as well as new approaches that have appeared and developed practically simultaneously with Google Earth, but significantly different from it.

Albert Gore's definition revealed two key properties of the Digital Earth that can be used to classify the variety of geospatial products: 1) multi-resolution representation, and 2) three-dimensional representation. In fact, all geographic maps are confined inevitably to a certain scale and projection. Both these confinements are fundamental and determined by the basic cartographic principle. Throughout history, humankind has dealt only with geographic information confined to a strictly defined scale and making sense only at that scale. At the same time, this restriction was perceived as temporary and forced consequence of technical imperfection. Its overcoming in the future was envisioned, expected and perceived as achievement of the magic horizon of geography development.

Now this future has become a reality. The Digital Earth is not confined to just one scale and one projection. For example, in Google Earth the user can change smoothly and interactively both the view angle of the planet as a whole or the selected scene, and the distance to it by means of an extremely simple and natural interface. The existence of two polar geographic products - maps (confined to projection and scale) and the Digital Earth (free of these limitations) - makes it possible to use the degree of freedom in terms of scale and viewing angle as classification criteria to systematize the whole variety of geographic products.

A simple confinement-based analysis unveils four possible types of basic global geospatial visualizations to be identified (Fig. 1): 1) maps and GIS (regardless of the 
type of their media) that incorporate the cartographic principle and confined to specific scale and specific projection; 2) globes that allow viewing the scene from any perspective from the upper hemisphere, but do not allow changing the scale; 3) atlases and geoportals (cartographic-based solutions that allow selecting the required scale from a discrete set, but do not allow changing the projection); and 4) Digital Earth, completely free from restrictions of any kind in user's choice of projection or scale. The visible representation of the features of these four types can be obtained with the help of "Angle-Distance" diagrams [16]. Obviously, it is possible to create mixed products that combine the properties of the first three types. At the same time, the Digital Earth cannot be combined with other types of geo-visualizations because it is comprehensive and contains all of them.

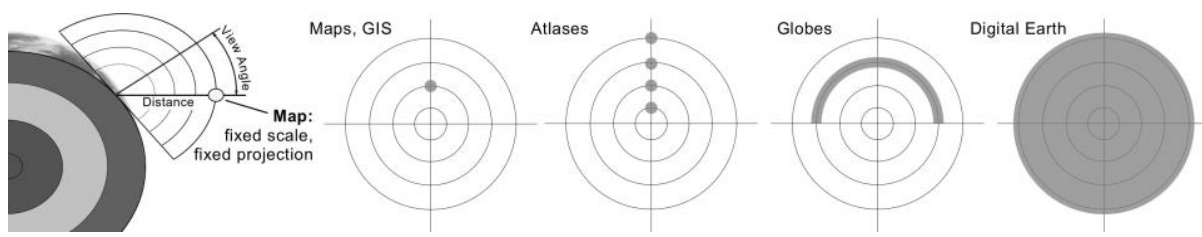

Fig. 1. "Angle-Distance" diagram and four basic types of geospatial visualizations.

How should we approach the definition of the Digital Earth based on the proposed classification? It seems natural to use and clarify the existing definition for this purpose. In doing so, it should be borne in mind that the definition must comply with generally accepted rules of logic [17]:

1. definition should state the essential attributes of the species;

2. definition must not be circular;

3. definition must be neither too broad nor too narrow;

4. ambiguous, obscure, or figurative language must not be used in a definition;

5. definition should not be negative when it can be affirmative.

To create a definition of the Digital Earth in accordance with these rules let's consider the basic definition proposed by Gore in 1998 [1]. It consists of six elements that expresses different properties and features of the Digital Earth (see Fig.2). Accordingly, it is possible to reveal the meaning of these properties from today's perspective, taking into account the experience of the first applications in which the principle of Digital Earth was embodied.

$$
\begin{aligned}
& \text { I believe we } \mid \text { need a "Digital Earth". A } \mid \text { multi-resolution, } \\
& \text { three-dimensional representation of the planet, into which } \\
& \text { we can embed vast quantities of geo-referenced data. } \\
& \text { Al Gore, } 1998
\end{aligned}
$$

Fig. 2. Elements of 1998 Albert Gore's definition of Digital Earth to be reviewed, clarified and refined if necessary. 
1. Digital. The adjective "digital" can refer both to a special type of signs, and their specific implementation in the computer systems. Here the Digital Earth is clearly associated with "computer" technologies, which narrows down the meaning of this term. However, there is no reason to reject the possibility of realizing it through any other technology. Moreover, the Earth itself, as a phenomenon that matching the definition of the "Digital Earth", is not actually a "digital" phenomenon. Moreover, today all information systems are "digital" in a computer term, and all information systems ever known are "digital" in semiotics term. Accordingly, the adjective "digital" should be regarded as a metaphor for novelty, but not as an essential property of the Digital Earth.

2. Multi-resolution. Multi-resolution refers to the presence of several different resolutions. However, the capabilities of the Digital Earth are incomparably wider - it contains an infinite number of resolutions, allowing for continuous and interactive zooming by the user [18]. The ability to provide continuous zooming is the most impressive feature of the Digital Earth. Therefore, we can say that in the case of the Digital Earth, we are not dealing with a multiresolution, but rather with a scale-less approach.

3. Three-dimensional. 3D-representation means ability to change the direction of sight smoothly and interactively beyond the limited number of the cartographic projections and even generate absolutely impossible, prohibited by cartography direction of sight - for example, from bottom to top. Threedimensional representation is implemented in the Digital Earth indeed. In fact, ability to generate any possible direction of sight by user "mirrored" scale-less property of the real Earth. Therefore, we can say that a projectionless approach is implemented in the Digital Earth.

4. Representation. Indeed, the Digital Earth as a model (or twin, or replica, or copy) of the Earth could be regarded as a representation. Moreover, this representation does not confined to specific scale and projection and does not require inevitable and irreversible reducing the raw data to specific scale or projection.

5. Planet. In the common cartography "representation of the planet" means representation of the surface of the planet. Instead, the Digital Earth does not limited by the surface itself. It helps to place and visualize any objects, data, processes, etc. within geocentric space around the Earth - like satellite constellations, urban canyons, terrain, seabed, cloud formations, etc. Therefore, the Digital Earth is geocentric model rather than representation of the planet's surface like map.

6. Vast quantities of geo-referenced data. Indeed, the Digital Earth ables to collect vast quantities of geo-referenced data. But now any hypertext-based information system ables to collect same quantities of the same data. The striking and unique property of the Digital Earth is an ability to provide rich, contiguous context for any data of any scale (within wide range of accessible scales), any extent and any direction of sight within geocentric volume that includes even satellite constellations.

The striking properties of the Digital Earth look quite obvious and natural, because they mimic the properties of the Earth itself as it percepted by human. This 
summa of properties was forecasted far before nowadays as a miracle geographical system of the future [19]. Thus, the question arises - what made it possible to achieve these obvious properties in the Digital Earth, but not with other geographical instruments?

Albert Gore directly emphasized in his speech that the important or even main feature of the Digital Earth is the use of images instead of presenting information with the help of signs: "We have long known that we have trouble remembering more than seven pieces of data in our short-term memory. That's a low bit rate. On the other hand, we can absorb billions of bits of information instantly if they are arrayed in a recognizable pattern within which each bit gains meaning in relation to all the others" [1]. This statement directly declares that low efficiency of perception of information is connected with its transmission by means of signs, while much higher efficiency is reached at use of unsigned tools of information transmission - visual images and, more broadly, direct sensual perception. The Digital Earth was the first geographic system that showed the advantage of images over cartographic signs in visualizing the geospatial environment. Avoiding signs as the primary carrier of geospatial information is a key feature of the Digital Earth and ultimate factor of its novelty.

Therefore, we could propose the modified version of the definition of the Digital Earth:

Definition 1. Digital Earth is a scale-less and projection-less geospatial visualization of geocentric volume that similar to a real one by the means of incorporating the signless representation of the geospatial context.

The proposed definition is largely empirical because it combines the theoretical prediction of the Digital Earth with the experience of the usage of the applications inspired by this concept. As a result, this definition allows a critical discussion about possibilities of the Digital Earth, its relationships with different approaches to geovisualization, and contains significant potential for further interdisciplinary research. Moreover, this definition includes the vision of the typology of the geospatial visualizations, therefore it makes possible comparative study of the different approaches for geospatial visualization.

Use of images instead of cartographic signs to represent the geospatial context is a significant challenge to classical semiotics, which assumes that all information media must be treated exclusively as signs. Under this approach, all images, such as iconic images, are supposed to be classified as the most primitive so-called "iconic signs", while the Digital Earth is to be interpreted as a return from complex and "mature" signs to more primitive signs.

An alternative point of view indicates that attributing all images, including those obtained by remote sensing means, to iconic signs generates at least two logical fallacies.

1. It leads to violation of "law of parsimony".

2. It contradicts the idea of artificial nature of signs and, therefore, their historical development.

In order to resolve a crisis, it must be assumed that information could be transmitted not only by signs but also by other entities that were not signs. It had been sug- 


\section{E. Eremchenko}

gested that they should be called "zero signs" [20], because their role in semiotics was similar to that of zero in mathematics.

In this case, the Digital Earth should be considered a heterogeneous information system that uses both unsigned media and usual signs to achieve effective perception of the environment. The "zero sign hypothesis" needs to be critically considered, and the concept of the Digital Earth itself needs to be studied from a semiotic perspective.

Above considerations allow to separate Digital Earth and its alternatives.

\section{Discussion}

The use of degrees of spatial freedom for classification of geospatial visualizations allows to reveal four definite types which fit the internal logic of the historical development of the geospatial method. All three alternative modes of geospatial visualization: 1) maps, atlases and GIS, 2) geoportals, 3) globes can be fully incorporated into the Digital Earth; otherwise is impossible. A factor that provides a new quality of perception in the Digital Earth mode is the use of images instead of signs as the primary carriers of geospatial context. Any of the four types of geo- visualizations allows to integrate the vast amount of information due to using of hypertext addressing mechanism, however the quality of its perception will be different. Digital Earth, due to its rich spatial context is possible to ensure the unrestricted perception of any spatial information in an extremely wide and not limited range of scales and in all possible diversity of directions of sight.

All geospatial approaches need raw information and the means to obtain it. All these methods are non-specific and can be used equally (with varying efficiencies) in any of the geospatial approaches. Therefore, data acquisition tools and related systems (geodesy and navigation, remote sensing, crowdsourcing, data processing capabilities, interfaces, etc.) are not the inherent components of the Digital Earth. Instead, Digital Earth will impact these variety of geospatial means and tools, significantly changing the ways for data capturing, processing and utilization. Also architecture and configuration of these systems will vary greatly accordingly with the requirements of new geospatial paradigm.

The same considerations apply to the analysis of the type of information media used in the Digital Earth and its analogues. The definition of "digital", which was a symbol of novelty back in the 1990s, has lost its original meaning by the second decade of the 21st century, since nowadays all information systems have completely or mainly migrated to the Internet ecosystem and are presented exclusively as computer ("digital") carriers. Nevertheless, the Digital Earth retains its exclusivity, and no other method of geo-visualization, also implemented through digital technologies, can match it in its capabilities. This transformation has not affected the essence of the information systems themselves - they were and still are based on the same signs as those developed centuries and millennia before the Internet. The revolutionary nature of the change is due to the fact that, for the first time in history, the Internet has enabled the free circulation of images constructed with the same "digital" signals as an usial text signs and digits, but simulating direct, non-signatory perception with increasing accuracy. The determinant of "Digital" in the name Digital Earth is historical - it would be more accurate to call it "Sign-less Earth" instead the "Digital Earth". 
The relationship between the Digital Earth, its counterparts and supplementary systems is shown in Figure 3.

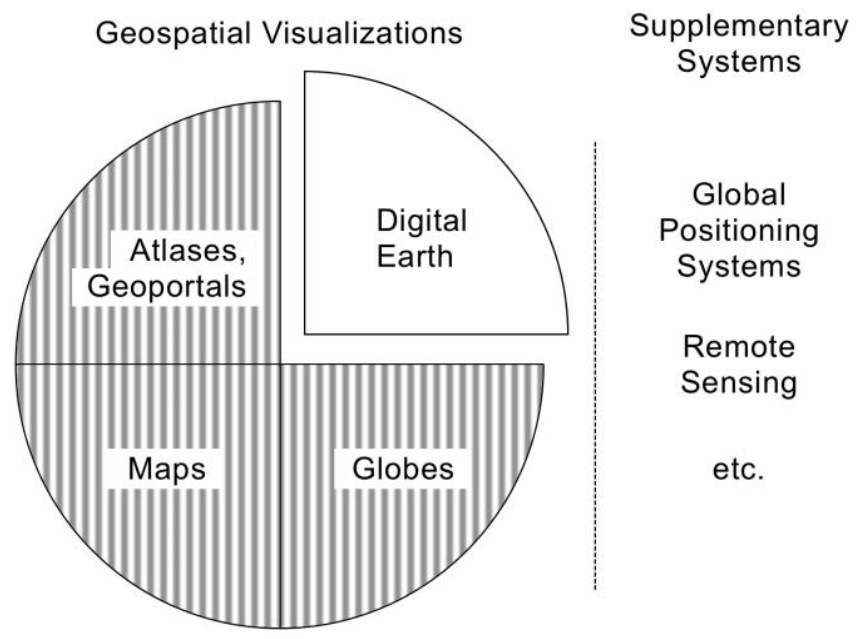

Fig.3. Types of geospatial visualizations and supplementary systems. Non-Digital Earth geospatial approaches are dashed.

\section{Conclusions}

The Digital Earth is one of the four basic types of geospatial visualizations. All other types of geospatial visualizations - maps, atlases, globes - cannot be considered as the Digital Earth. Means of gathering geospatial information and other complementary systems like GNSS, remote sensing and communication satellites etc., cannot be attributed to the Digital Earth in the same way as well.

Digital Earth as a new approach to working with geospatial information is radically different from the other three, but uses the same sources of information - although its usage becomes completely different. For example, recreating a sign-less geospatial context requires a very different implementation of remote sensing orbital constellations. The architecture of control systems is changing, as the elimination of largescale image differentiation eliminates the only need for their hierarchical construction.

The Digital Earth no longer fits the paradigm of classic cartography. That is why understanding the nature of the Digital Earth, forming its scientific definition and internal development of the Digital Earth as an interdisciplinary scientific paradigm and relevant methods and technologies are so important.

Also should be noted some unresolved issues:

1. Development of methods for visualization of solid media with a complex internal structure. This is necessary in order to extend the Digital Earth to represent areas that cannot be visualized by conventional means - the geology of Earth, processes in the atmosphere and outer space, etc. In fact interior of the Earth is the only "white spot" remaining in the modern Digital Earth. 


\section{E. Eremchenko}

2. To date, there is no Digital Earth standard that describes its minimum set of functions without regard to technical facilities and platforms, exchange formats, etc. The development of such a standard is extremely important to ensure the systemic development of the Digital Earth.

3. The Digital Earth was the first information system to use unsigned tools to represent geospatial context. The historical tradition, according to which signs were the only possible means of information transmission, and the development of increasingly complex sign systems, the most important means of social development, has reversed. The consequences of this turn for society have yet to be studied and understood.

4. Digital Earth is an unmatched tool for seamless consolidation of heterogeneous social information - for example, medical, biological, environmental, etc. The development and integration of effective tools for handling social geospatial information in the Digital Earth environment and the adaptation of management structures to this new tool for sustainable development is an urgent task.

\section{References}

1. Gore A.: The Digital Earth: Understanding our planet in the 21st Century, http://The_Digital_Earth_Understanding_our_planet_in_the_21st_Century.doc last accessed 2020/07/21.

2. GoogleEarth, https://www.earth.google.com, last accessed 2020/06/12.

3. More than one billion downloads for Google Earth, https://www.gearthblog.com/blog/archives/2011/10/more_than_one_billion_downloads_fo r.html, last accessed 2020/06/15.

4. About ISDE, http://www.digitalearth-isde.org/society/54, last accessed 2020/06/12.

5. Symposium, http://www.digitalearth-isde.org/symposium, last accessed 2020/06/12.

6. Summit, http://www.digitalearth-isde.org/summit, last accessed 2020/06/12.

7. International Journal of Digital Earth, http://www.digitalearth-isde.org/publication/70, last accessed 2020/06/12.

8. Big Earth Data, http://www.digitalearth-isde.org/page/836, last accessed 2020/06/12

9. Digital Earth Australia, http://www.digitalearth-isde.org/news/805, last accessed 2020/06/12.

10. Digital Earth implemented in Russia, http://www.digitalearth-isde.org/news/810, last accessed 2020/06/12.

11. Geoscience Australia to stop printing and selling topographic maps from December, https://www.abc.net.au/news/2019-10-03/geoscience-australia-stop-printing-and-sellingtopographic-maps/11566698, last accessed 2020/06/12.

12. Guo, H., Goodchild, M., Annoni, A. (eds): Manual of Digital Earth. Springer, Singapore. (2020).

13. Sad Lack of Visible Progress on Google Earth for Chrome and Mobile One Year Later, https://www.gearthblog.com, last accessed 2020/06/12.

14. Digital Earth Vision 2020, Workshop, http://www.digitalearth-isde.org/ssw/147, last accessed 2020/06/12.

15. Bulgakov, M.: Master and Margarita. Transl. by Glenny, M. Collins and Harvill Press. London (1967). 
16. Baturin, Yu., Eremchenko, E., Zakharova, M.: 3D-document and Digital Earth. In: CEUR Workshop Proceedings 2485, pp. 155-158, Bryansk (2019).

17. Copi, I., Cohen, C., McMahon, K.: Introduction to Logic. Routledge, NY (1982).

18. Li, Z., Yan, H.: Transformation in Scale for Continuous Zooming. In: Guo, H., Goodchild, M., Annoni, A. (eds) Manual of Digital Earth. Springer, Singapore. (2020).

19. Eremchenko, E.: Prehistory of the Digital Earth Concept (Russian). Geocontext 7(1), 4453. (2019).

20. Eremchenko, E.: Visualization and new definition of sign. In: GraphiCon 2018 - 28th International Conference on Computer Graphics and Vision, pp. 301-303. TPU, Tomsk (2018). 